\title{
En-bloc excision of Giant Cell Tumors of the distal radius and arthrodesis of the translocated ipsilateral ulna: A report of two cases
}

\author{
Eva Campos-Pereira ${ }^{\mathrm{a},{ }^{*}}$, João Vale ${ }^{\mathrm{b}}$, Tiago Amorim-Barbosa ${ }^{\mathrm{b}}$, Filipe Rodrigues ${ }^{\mathrm{b}}$, Arnaldo Sousa $^{\mathrm{b}}$, \\ Pedro Teixeira Mota ${ }^{b}$, Vânia Oliveira ${ }^{b}$, Alexandre Pereira ${ }^{b}$, Pedro Cardoso ${ }^{\text {b }}$
}

${ }^{a}$ Department of Orthopaedics, Hospital Central do Funchal, Funchal, Portugal.

${ }^{b}$ Department of Orthopaedics, Centro Hospitalar e Universitário do Porto, Porto, Portugal.

\begin{abstract}
The distal radius is the third most common site of giant cell tumor of bone (GCTB). The local aggressive invasion of this rare neoplasm requires reconstructive solutions after wide excision. The authors present two cases of patients diagnosed with Campanacci grade III GCTB of the distal radius successfully treated with en-bloc excision and translocation of the ipsilateral ulna. Pre-operative application of denosumab was given for one year to both patients. At one year of follow-up, both patients are disease-free and reported satisfactory results on Quick - Disabilities of the Arm, Shoulder and Hand (Quick-DASH) questionnaire and modified Musculoskeletal Tumor Society (MSTS) score. Although a challenge, the reported procedure offers good oncological and functional outcomes.

Keywords: Giant cell tumor of bone; distal radius; en-bloc excision; translocation; ipsilateral ulna; wrist arthrodesis
\end{abstract}

\section{INTRODUCTION}

Giant cell tumor of bone (GCTB) is a benign but locally aggressive intramedullary bone tumor that was first described in 1818 by Cooper and Travers ${ }^{[1-3]}$. The distal radius is the third most common site of GCTB after distal femur and proximal tibia ${ }^{[1,4-7]}$. Local recurrences occur in $70 \%$ of the cases within 24 months after excision and lung metastasis in $2 \%$ of the patients ${ }^{[7-10]}$.

Typically, local pain with an increased swelling is reported and a pathological fracture occurs in $10 \%$ of the patients because of the tumor's osteolytic nature ${ }^{[3,11]}$. The radiological system of 3 stages described by Campanacci et al. demonstrates a clinical-radiological cor-

* Correspondence to: Eva Campos-Pereira

Mailing address: Department of orthopaedics, Hospital Central do Funchal, Funchal, Portugal.

E-mail: eva.campos.pereira@gmail.com

Received: 02 September 2021 / Accepted: 15 December 2021 relation. In stage I, latent lesions have a well-marginated border and do not perforate the cortical bone. Stage II lesions are active, without a radiopaque rim or soft tissue involvement. Stage III tumors are aggressive, without well-defined limits, with rapid and permeated growth, with involvement of the surrounding soft tissue. For both stages, I and stage II lesions, intralesional curettage and acrylic bone cement (or other substitutes) are the most reported treatment ${ }^{[3-8,10,12-14]}$. Nevertheless, more aggressive treatment protocols are required to prevent the recurrence of lesions at stage III. Although adjuvant agents decrease the recurrence rates of intralesional curettage, this primary option has higher recurrence rates (up to 27\%) than wide resection (0 to $12 \%)^{[6,8,15,16]}$.

Denosumab, a human monoclonal antibody against RANKL (receptor activator of nuclear factor-kB ligand), contains tumor growth and facilitates resection without contamination when used as neoadjuvant therapy in aggressive lesions ${ }^{[1,10]}$. 
After resection of complex lesions, surgeons must consider the pros and cons of the different reconstructive options already described. Despite the consensus in radical tumor excision, the best wrist reconstructive option is not well defined.

The authors describe two cases of patients diagnosed with Campanacci grade III GCTB of the distal radius reconstructed with ipsilateral ulnar autograft and wrist fusion following en-bloc resection with good oncological and functional results.

\section{CASE REPORT}

\section{Case 1}

A 35-year-old right-hand dominant male presented with a painful and enlarging mass on his left wrist, with six months evolution. The patient referred that, two months before mass detection, a pathological

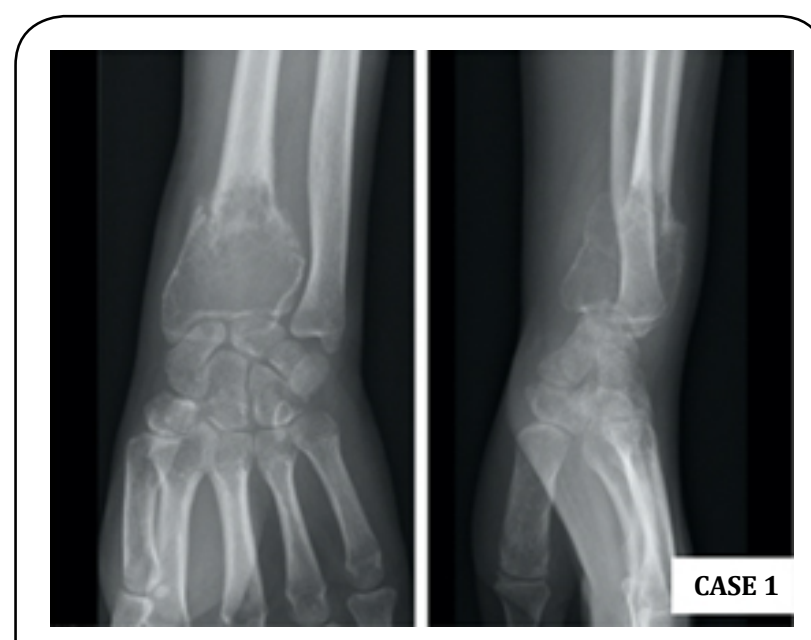

Figure 1. Anteroposterior (AP) and lateral radiographs revealing pathological fracture after minor trauma.

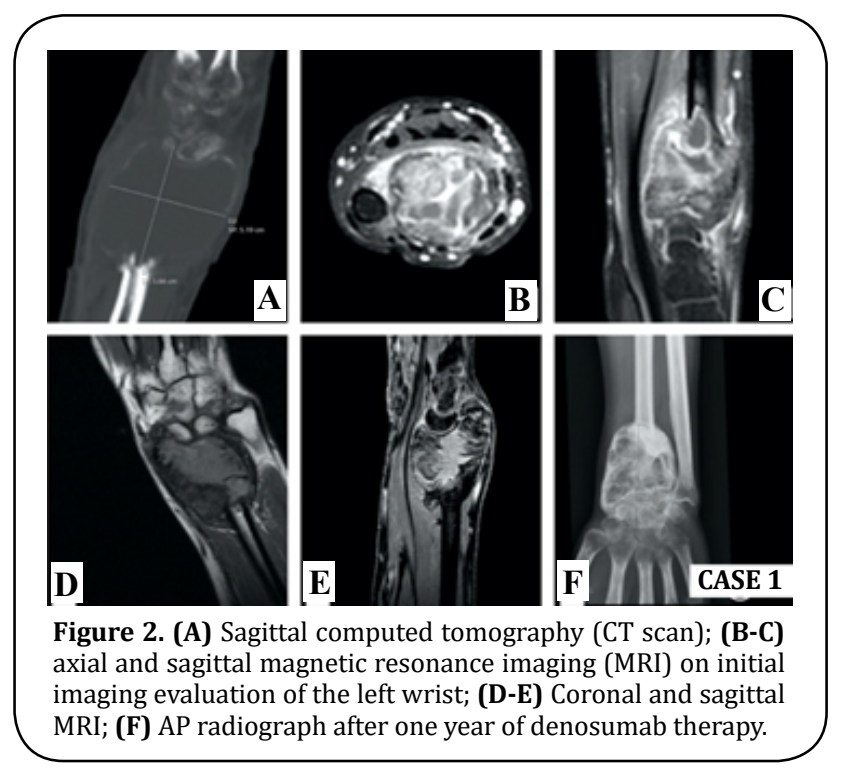

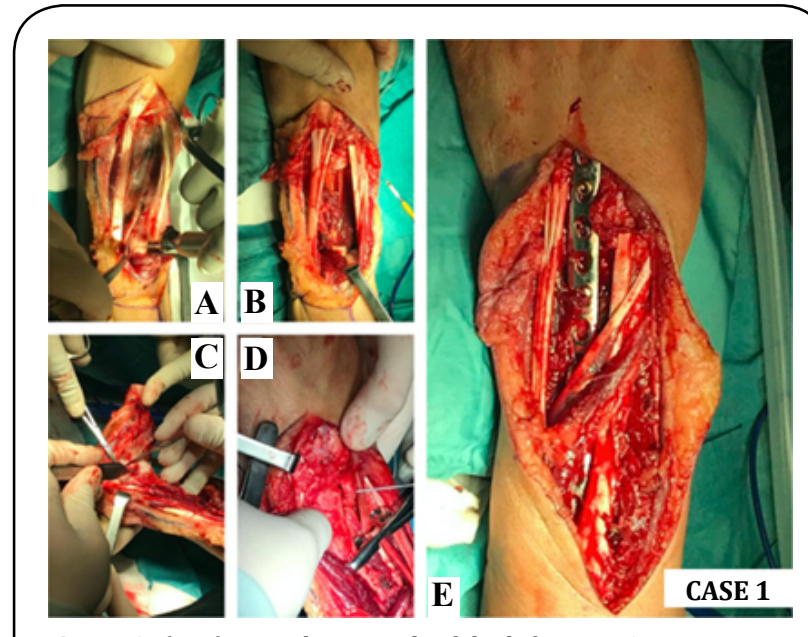

Figure 3. (A-B) Dorsal approach of the left wrist: 8 centimeters distal radius osteotomy and en-bloc resection; (C-D) after lunate removal and osteotomy of the distal ulna, translocation of the ulnar fragment; (E) wrist arthrodesis using a $3.5 \mathrm{~mm}$ locking compression plate (LCP).

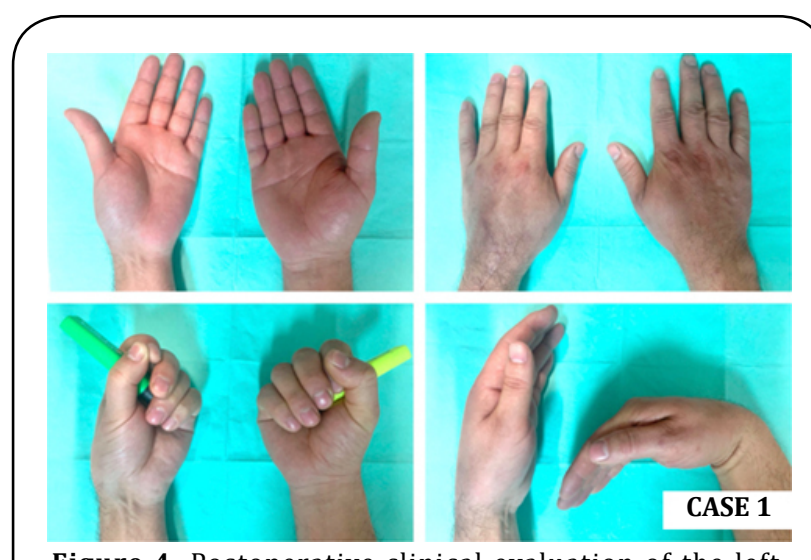

Figure 4. Postoperative clinical evaluation of the left operated wrist at one year of follow-up, compared with the contralateral side.

fracture was diagnosed after minor trauma (Figure 1). On physical examination the distal forearm was tender and hypoesthesia in the ulnar nerve territory was present. Pre-operative anteroposterior and lateral radiographs, computed tomography scan (CT scan), and magnetic resonance imaging (MRI) revealed an expansible lesion located within the epiphysis of the distal radius, compatible with a Campanacci grade III GCTB (Figure 2). He underwent a CT scan guided core biopsy. The histological report confirmed GCTB. No other lesions were detected in staging imaging. The patient completed one year of neoadjuvant therapy with denosumab (120 mg subcutaneous (SC) per 28 days, during 12 months) (Figure 2). The patient underwent an 8 centimeters dorsal en-bloc resection of the distal radius and an ulnar osteotomy at the same level of the radius osteotomy, retaining muscular attachments. The lunate was removed and the joint surface of the ulna as 
well as the remaining carpal bones was decorticated. The distal ulna was transposed and aligned with the remaining radius and the third metacarpal bone. An uneventful fixation was performed with a dorsal 3.5 $\mathrm{mm}$ locking compression plate (LCP) with slight dorsiflexion and ulnar deviation (Figure 3). The histological report confirmed wide resection (R0).

The patient was immobilized with an above-elbow cast for three weeks and a below-elbow splint for two more weeks. The patient underwent an intensive program of physiotherapy for eight months. At one year of follow-up, the patient presented full flexion/extension of the fingers and full supination/pronation (SN/PN) (Figure 4) without pain. The modified Musculoskeletal Tumor Society score (MSTS) was 21 (compared to the pre-operative value of 9) and the Quick - Disabilities of the Arm, Shoulder and Hand questionnaire (QuickDASH) was 22,7 (compared to the pre-operative value of 88,6$)$. The mean handgrip value, evaluated with a Jamar dynamometer, for the operated side was 51,8 Libras (lbs) compared to 93,9 lbs for the contralateral side. Radiographs showed bone fusion at both ends of

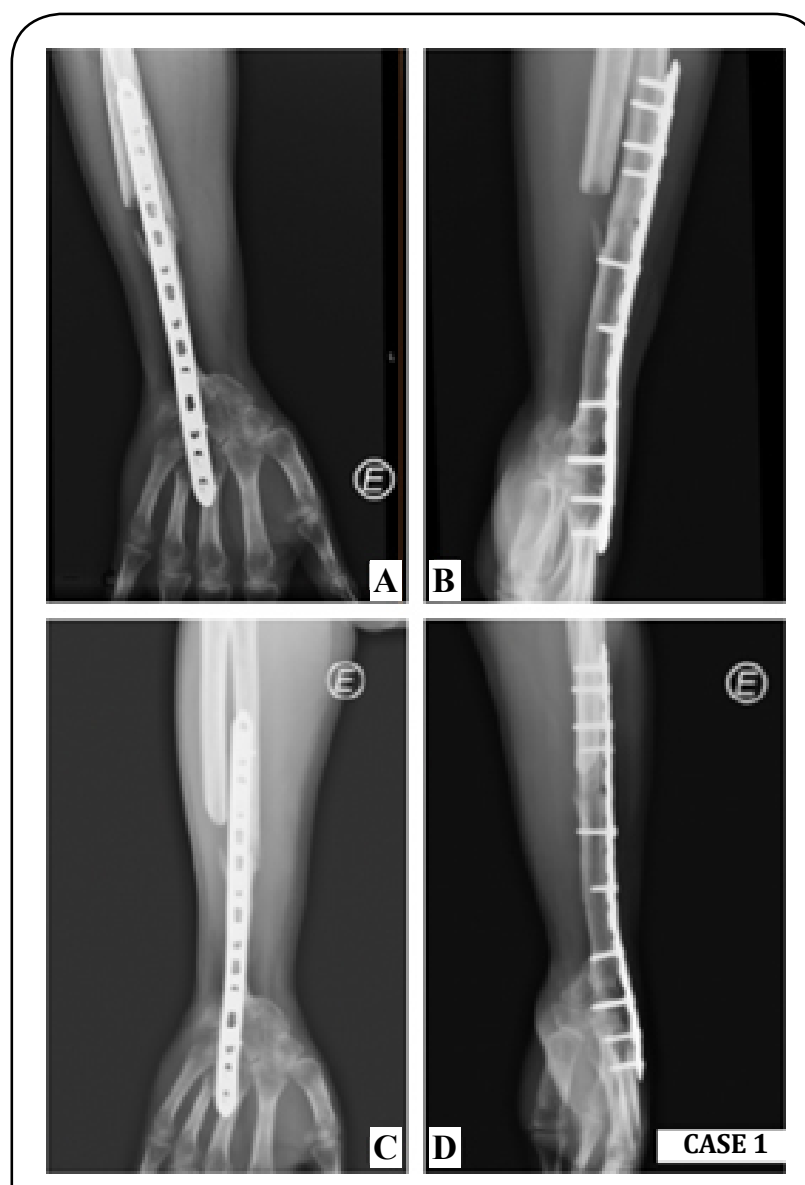

Figure 5. (A-B) AP and lateral radiographs at one year of follow up; (C-D) AP and lateral radiographs at two years of follow up; both showing signs of union without local recurrence. the ulna and discarded local recurrence at two years of follow-up (Figure 5).

Case 2

A 25-year-old right-hand dominant female presented with right wrist pain over an enlarging mass for one year. The patient reported a gradual increase in size over the last two months. On physical examination, the dorsal distal radius was tender and wrist motion was painful. The X-ray and CT scan revealed an expanded osteolytic lesion. MRI detected a huge osteolytic lesion and soft tissue extension with $4 \times 3,3 \times 2,3$ $\mathrm{cm}$, compatible with a Campanacci grade III GCTB (Figure 6). Staging imaging excluded other lesions. The histology report of the imaging-guided core biopsy showed a GCTB. After one year of denosumab therapy (120 mg SC per 28 days, during 12 months) (Figure 6) the patient underwent the same surgery as in Case 1. Wrist arthrodesis with a $3.5 \mathrm{~mm}$ LCP plate was performed after a $6 \mathrm{~cm}$ dorsal en-bloc resection of the distal radius and translocation of the ipsilateral ulna (Figure 7). There were no major complications related to the procedure. The histological report confirmed complete resection with free margins. An above-elbow cast was applied for three weeks, after which a belowelbow was used for two more weeks. After two months the patient had a full SN/PN and after six months the patient was able to perform the daily activities without restrictions or pain (Figure 8). At six months the MSTS score was 24 (compared to the pre-operative value of 19), the mean handgrip strength value for the operated side was $33,1 \mathrm{lbs}$ compared to $49,8 \mathrm{lbs}$ for

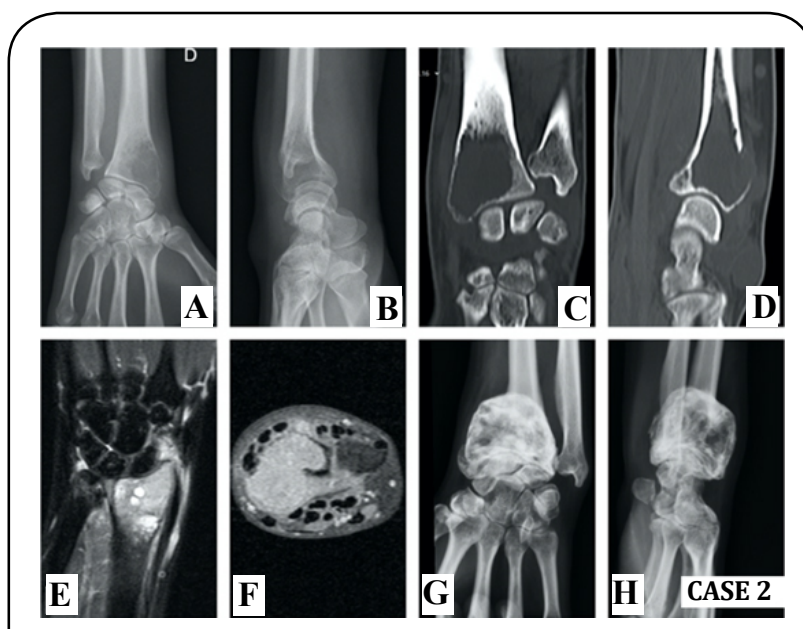

Figure 6. (A-B) AP and lateral radiographs, coronal and sagittal CT scans (C-D) and coronal and axial MRI (E-F) of the right wrist during primary imaging evaluation; (G-H) Preoperative radiographs of the distal right wrist after one year of denosumab therapy. 
the non-operated side, and scored 27,3 in Quick-DASH questionnaire (the same value as in the pre-operative assessment). At two years of follow-up, the patient has no clinical or imaging signs of local recurrence (Figure 9).

\section{DISCUSSION}

GCTB of GCTB of the distal radius is the third most common site of this relatively rare neoplasm, corresponding to $10 \%$ of all cases ${ }^{[2,4-7,17,18]}$. Despite its benign label, the distal radius is the most common primary site responsible for metastases and a high focus on ruling out pulmonary metastasis is mandatory in the pre-operative assessment ${ }^{[9,10,12,19]}$.

In well-marginated cortical borders, curettage with bone grafting/cement packing is acceptable despite the recurrence rates up to $50 \%{ }^{[4,5,7,17,19]}$. Campanacci

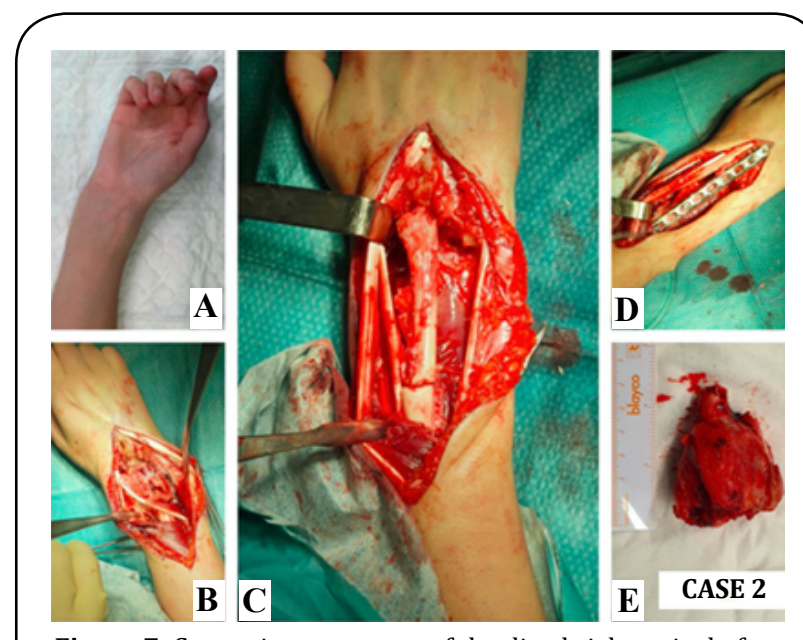

Figure 7. Cosmetic appearance of the distal right wrist before surgery (A) and dorsal approach (B); translocation of the distal ulna after distal radius osteotomy (C) and dorsal wrist arthrodesis with a $3.5 \mathrm{~mm}$ LCP plate (D); en-bloc resection of $6 \mathrm{~cm}$ distal radius (E).

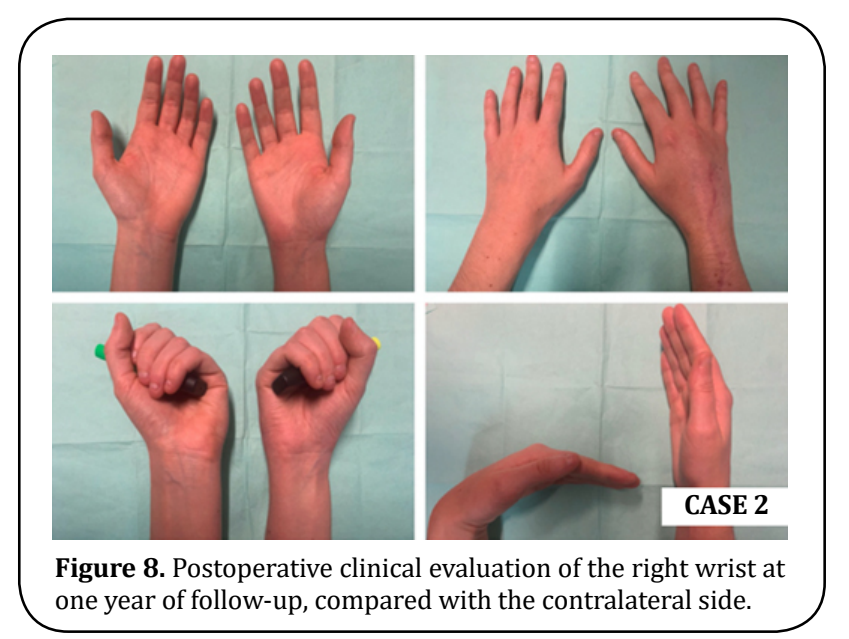

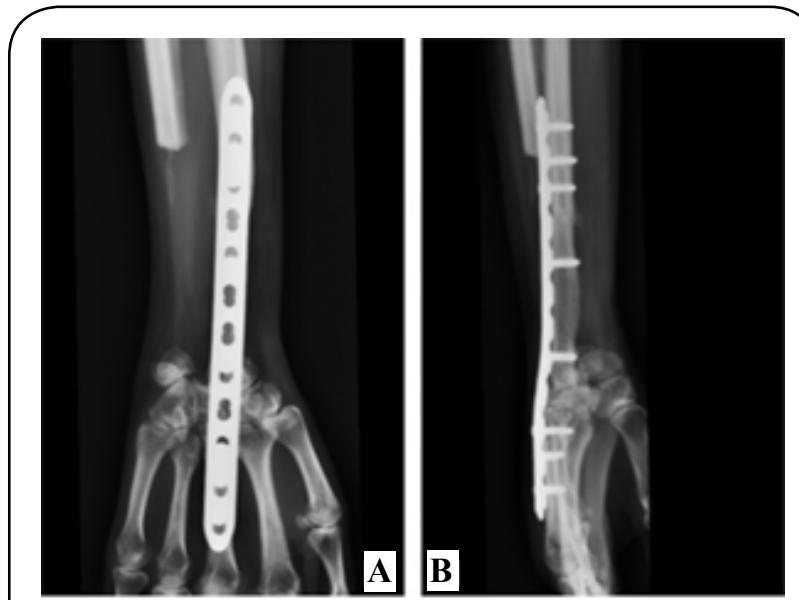

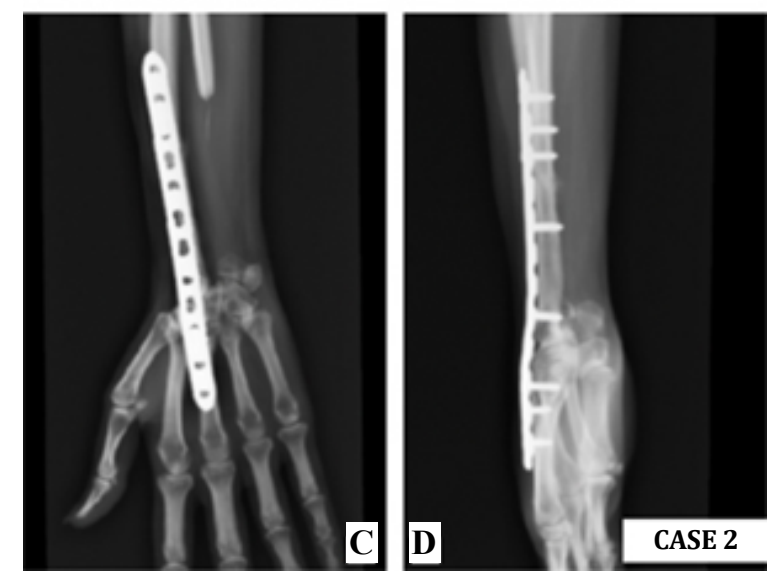

Figure 9. (A-B) AP and lateral radiographs at one year of follow-up; (C-D) AP and lateral radiographs at two years of follow-up; both excluding local recurrence.

grade, pathological fractures, tumor site, and adjuvant therapy are recognized predictors of local recurrence ${ }^{[1,}$ ${ }^{19]}$. Studies have correlated the incidence of metastases with aggressive growth and local recurrence ${ }^{[20,21]}$.

The treatment of the rare Campanacci III lesions is a much more arduous task. Wide resection must be performed to avoid recurrence and reconstructive options should be considered to preserve wrist function. The en-bloc resection is widely acceptable in expansive lesions, recurrent tumors, and when the articular surface is largely damaged or collapsed $[17,18,22]$. Due to the proximity with other bones (ulna and carpal bones) and other soft tissue structures, intralesional excisions, even when adjuvant therapies are used, have poor local control when compared to enbloc resection ${ }^{[1,2]}$. Reconstruction of the wrist by ulnar translocation after total resection was first described in 1982 by Seradge ${ }^{[5,6,19,22]}$ and several techniques to fixate the transposed ulna were described: Steinmann pins, T-shape plates, dynamic compression plates, cloverleaf plates, and K-wires ${ }^{[19]}$. The ipsilateral ulnar translocation technique is a surgical procedure that 
avoids donor site morbidity and allows an adequate muscular cover with improved vascularity $[5,7,9$, ${ }^{17]}$. Furthermore, the option of a single bone in the forearm avoids the complication of ulnar variance when other reconstruction techniques - fibular, iliac crest, allografts - are considered ${ }^{[6]}$. When compared with the proximal vascularized fibula, the ipsilateral autologous ulna does not require microsurgical skills ${ }^{[7,17]}$. Autologous ulna also eliminates the risk of graft rejection ${ }^{[5]}$. Comparing all the fixation methods of the translocated ulna, Chobpenthai et al. ${ }^{[19]}$. concluded that distal radius plating is the less traumatic technique and achieves the best functional and cosmetic results.

Despite the flaws pointed out to this technique (poor vascularity, proximal and distal nonunion, and lack of motion), arthrodesis results in less post-operative pain and good to excellent results in grip strength ${ }^{[15}$, ${ }^{19]}$. The most accepted wrist position after fusion is $10^{\circ}$ dorsiflexion and 5-10 ${ }^{\circ}$ ulnar deviation ${ }^{[9]}$.

Another possibility for reconstruction could be a custom-made mega prosthesis to preserve some motion in the sagittal e coronal planes. However, the literature is scarce and these patients were too young and with some functional demand, so the arthroplasty would be quickly condemned to fail ${ }^{[23]}$.

No less important is the role of denosumab, used as neoadjuvant therapy, in making tumor dissection viable. The massive cortical destruction and friable nature of GCTB benefit from this chemotherapeutic agent in reducing pain and suppressing the tumor ${ }^{[1,10]}$. In conclusion, although treatment of Campanacci grade III GCTB of the distal radius remains a challenge, the combination of neoadjuvant denosumab therapy and tumor en-bloc resection have a good prognosis. The reported reconstructive technique achieved good functional and oncological outcomes.

\section{DECLARATIONS}

\section{Acknowledgments}

Work performed at Orthopaedics Department of Centro Hospitalar e Universitário do Porto, Porto, Portugal. The authors would like to thank Luís Oliveira for his help in the preparation of the manuscript.

\section{Author's contributions}

All the authors contributed substantially to the design and production of this article.

Financial support and sponsorship
None.

\section{Conflicts of interest}

All authors declared that there are no conflicts of interest.

\section{Ethical approval and consent to participate}

This study, which included human samples was written informed consent was obtained from the patients for publication of these case reports and any accompanying images. All studies involving human subjects were in accordance with the Helsinki Declaration.

\section{REFERENCES}

1. Zou, C., Lin, T., Wang, B., Zhao, Z., Li, B., Xie, X., ... \& Shen, J. (2019). Managements of giant cell tumor within the distal radius: a retrospective study of 58 cases from a single center. Journal of bone oncology, 14, 100211.

2. Liu, Y.-p., Li, K.-h., \& Sun, B.-h. (2012). Which treatment is the best for giant cell tumors of the distal radius? A meta-analysis. Clinical Orthopaedics and Related Research, 470(10), 2886-2894.

3. Sánchez-Torres, L., de la Parra-Márquez, M., CruzEscalante, A., Ramírez-Barroso, R., \& EspinozaVelazco, A. (2017). Microsurgical reconstruction in limb salvage due to a giant cell tumor of the distal radius. Case report. Acta ortopédica mexicana, 31(2), 98-102.

4. Wang, Y., Min, L., Lu, M., Zhou, Y., Wang, J., Zhang, Y., ... \& Duan, H. (2020). The functional outcomes and complications of different reconstruction methods for Giant cell tumor of the distal radius: comparison of Osteoarticular allograft and three-dimensional-printed prosthesis. BMC musculoskeletal disorders, 21(1), 1-15.

5. Salunke, A. A., Shah, J., Warikoo, V., Chakraborty, A., Pokharkar, H., Chen, Y., ... \& Pandit, J. (2017). Giant cell tumor of distal radius treated with ulnar translocation and wrist arthrodesis: What are the functional outcomes? Journal of Orthopaedic Surgery, 25(1), 1-6.

6. Puri, A., Gulia, A., Agarwal, M., \& Reddy, K. (2010). Ulnar translocation after excision of a Campanacci grade-3 giant-cell tumour of the distal radius: an effective method of reconstruction. The Journal of bone and joint surgery British volume, 92(6), 875879. 
7. Ververidis, A. N., Drosos, G. I., Tilkeridis, K. E., \& Kazakos, K. I. (2015). Carpus translocation into the ipsilateral ulna for distal radius recurrence giant cell tumour: a case report and literature review. journal of orthopaedics, 12, S125-S129.

8. Xu, L., Jin, J., Hu, A., Xiong, J., Wang, D., Sun, Q., \& Wang, S. (2017). Soft tissue recurrence of giant cell tumor of the bone: prevalence and radiographic features. Journal of bone oncology, 9, 10-14.

9. Vyas, A., Patni, P., Saini, N., Sharma, R., Arora, V., \& Gupta, S. (2018). Retrospective analysis of giant cell tumor lower end radius treated with En bloc excision and translocation of ulna. Indian journal of orthopaedics, 52(1), 10-14.

10. Van Handel, A. C., Galvez, M. G., Brogan, D. M., Boyer, M. I., Cipriano, C. A., Hirbe, A. C., \& Pet, M. A. (2020). Vascularized Ulnar Transposition and Radioulnoscapholunate Fusion With Volar Locking Plate in a Dorsal Position Following Resection of Giant Cell Tumor of the Distal Radius. Techniques in hand \& upper extremity surgery, 24(3), 142150.

11. Lieberman, J. R. AAOS Comprehensive Orthopaedic Review 3: Lippincott Williams \& Wilkins; 2019.

12. Chalidis, B. E., \& Dimitriou, C. G. (2008). Modified ulnar translocation technique for the reconstruction of giant cell tumor of the distal radius. Orthopedics (Online), 31(6), 1.

13. Palacios, A. O., Ambite, J. C. M., \& Zbigniew. (2018). Giant Cell Tumors on the Fourth Metacarpal: about a case. Revista Iberoamericana de Cirugía de la Mano, 46, 40-45.

14. Cheng, C.-Y., Shih, H.-N., Hsu, K.-Y., \& Hsu, R. W.-W. (2001). Treatment of giant cell tumor of the distal radius. Clinical Orthopaedics and Related Research (1976-2007), 383, 221-228.

15. Zhang, J., Li, Y., Li, D., Xia, J., Li, S., Yu, S., ... \& Yang, Z. (2016). Clinical effects of three surgical approaches for a giant cell tumor of the distal radius and ulna. Molecular and clinical oncology, 5(5), 613-617.

16. Saikia, K. C., Bhattacharyya, T. D., Bhuyan, S.
K., Bordoloi, B., Durgia, B., \& Ahmed, F. (2011). Local recurrences after curettage and cementing in long bone giant cell tumor. Indian journal of orthopaedics, 45(2), 168-173.

17. Meena, D. K., Thalanki, S., \& Sharma, S. B. (2016). Wrist fusion through centralisation of the ulna for recurrent giant cell tumour of the distal radius. Journal of Orthopaedic Surgery, 24(1), 84-87.

18. Saini, R., Bali, K., Bachhal, V., Mootha, A. K., Dhillon, M. S., \& Gill, S. S. (2011). En bloc excision and autogenous fibular reconstruction for aggressive giant cell tumor of distal radius: a report of 12 cases and review of literature. Journal of orthopaedic surgery and research, 6(1), 1-9.

19. Chobpenthai, T., Thanindratarn, P., Phorkhar, T., \& Ingviya, T. (2020). The reconstruction after en-bloc resection of giant cell tumors at the distal radius: A systematic review and meta-analysis of the ulnar transposition reconstruction technique. Surgical Oncology, 34, 147-153.

20. Bertoni, F., Present, D., Sudanese, A., Baldini, N., Bacchini, P., \& Campanacci, M. (1988). Giant-cell tumor of bone with pulmonary metastases. Six case reports and a review of the literature. Clinical orthopaedics and related research, (237), 275285.

21. Siebenrock, K., Unni, K., \& Rock, M. (1998). Giantcell tumour of bone metastasising to the lungs: a long-term follow-up. The Journal of bone and joint surgery British volume, 80(1), 43-47.

22. McLean, J. M., Clayer, M., Stevenson, A. W., \& Samson, A. J. (2014). A modified ulnar translocation reconstruction technique for Campanacci grade 3 giant cell tumors of the distal radius using a clover leaf plate. Techniques in hand \& upper extremity surgery, 18(3), 135-142.

23. Hariri, A., Facca, S., Di Marco, A., \& Liverneaux, P. (2013). Massive wrist prosthesis for giant cell tumour of the distal radius: a case report with a 3-year follow-up. Orthopaedics \& Traumatology: Surgery \& Research, 99(5), 635-638. 